\title{
Dépérissement forestier et perturbations minérales aux niveaux histologique et cellulaire dans les aiguilles de Picea abies $L$. Étude par microanalyse $X$
}

\author{
JP Garrec 1, E Laitat 2, I Richardin 1, C Rose 1
}

1 INRA, Centre de recherches forestières de Nancy, Laboratoire d'étude de la pollution atmosphérique, 54280 Champenoux, France;

2 Faculté des sciences agronomiques de l'État, Département de biologie végétale, 5030 Gembloux, Belgique

(Reçu le 31 octobre1990; accepté le 6 mars 1991)

\begin{abstract}
Résumé - Des jaunisements foliaires liés à des carences minérales sont observés dans les arbres dépérissants. Pour comprendre les causes de ce phénomène et pour évaluer son impact physiologique, nous avons étudié dans des aiguilles vertes d'épicéas dépérissants, les concentrations en éléments minéraux aux niveaux histologique et cellulaire, grâce à l'utilisation d'une microsonde électronique. La microanalyse des différents éléments indique que globalement, dans les aiguilles, les effets des facteurs du dépérissement sont équivalents, sur la composition minérale, à un vieillissement accéléré de celles-ci. En effet on observe que ces facteurs, comme le vieillissement, entraînent en particulier une baisse de potassium et une augmentation de calcium et de manganèse dans les différents tissus. Cependant nous mettons en évidence, grâce à cette technique précise, que suivant les tissus le vieillissement ou les facteurs du dépérissement occasionnent des modifications ioniques différentes. Si les effets du vieillissement se manifestent surtout par des perturbations ioniques plus fortes au niveau des faisceaux libéro-ligneux, en revanche dans le cas de ce dépérissement, la baisse du potassium est particulièrement accentuée dans l'épiderme et les stomates. Parallèlement, nous détectons dans les complexes stomatiques une accumulation plus importante de calcium et de manganèse. Ces fortes variations ioniques, mises en évidence dans les tissus voisins de la surface des aiguilles d'épicéas dépérissants, suggèrent une participation non négligeable de paramètres atmosphériques (ozone, dépôts acides secs et humides) dans le phénomène du dépérissement. En particulier en perturbant le fonctionnement des stomates, ils pourraient être une des causes des déficits hydriques que plusieurs auteurs ont constaté dans les aiguilles.
\end{abstract}

Picea abies / dépérissement forestier / microanalyse $\mathrm{X}$ / élément minéral

Summary- Forest decline and ionic disturbances at the histological and cellular levels in the needles of Picea abies. An X-ray microanalysis study. Foliar yellowing in relation to mineral deficiencies has been observed in declining Norway spruce. To determine the causes of this phenomenon and to assess its physiological impact, the element contents at the histological and cytological level in green needles from declining spruce were studied by electron micro-probe. The microanalysis of different elements shows that at the whole needle level the effects of decline on the mineral content resemble premature senescence. Decline as well as ageing lead to a decrease in potassium and an increase in calcium and manganese in the different tissues. This accurate technique indicates that at the cellular level, ageing or decline cause different ionic disturbances. Ageing 
mainly leads to high ionic disturbances at the vascular tissue level; however decline results in high decrease in potassium in the epidermis and stomata. At the same time a greater accumulation of calcium and manganese is observed in the stomatal complexes. The high ionic disturbances detected in the tissues situated near the surface of needles from declining spruce, provide good evidence that atmospheric parameters are involved (ozone, wet or dry acid deposition) in the forest decline phenomenon. In particular, by disturbing the stomatal movements these ionic modifications might be one of the causes involved in the decrease in water content observed by several authors in these needles.

Picea abies / forest decline / $X$-ray microanalysis / mineral content

\section{INTRODUCTION}

Des phénomènes de dépérissement sont apparus vers 1970 dans un certain nombre de forêts européennes et, si la pollution atmosphérique (ozone et dépôts acides) est fortement soupçonnée d'intervenir dans ceux-ci, d'autres stress comme la sécheresse auraient un rôle primordial (Rehfuess, 1987; Becker, 1989; Roberts et al, 1989).

Ces phénomènes ont d'abord été observés sur des résineux, et se caractérisent visuellement et d'une façon assez générale par une chute des aiguilles et/ou des jaunissements de celles-ci.

De nombreuses analyses foliaires ont montré que ces jaunissements provenaient essentiellement de carences minérales qui concernaient surtout les éléments $\mathrm{Mg}, \mathrm{K}$ et $\mathrm{Ca}$ avec des importances relatives différentes selon les régions (Zoettl, Huettl, 1986; Landmann et al, 1987; Huettl, 1989).

Parallèlement, l'analyse des pluviolessivats sous couvert (throughfall et stemflow) a montré que généralement par rapport à la composition des pluies incidentes, ceuxci sont moins riches en $\mathrm{NH}_{4}$ et $\mathrm{NO}_{3}$ et plus concentrés en $\mathrm{K}, \mathrm{Ca}$ et $\mathrm{Mg}$, avec des variations selon l'espèce forestière et le site étudié (Lovett et al, 1985; Reynolds et al, 1989; Sigmon et al, 1989).

Plusieurs hypothèses ont été avancées pour expliquer ce dépérissement forestier.
Les carences minérales proviendraient d'un lessivage accéléré des ions de la feuille, avec pour conséquence une augmentation des concentrations en éléments dans les pluviolessivats. $\mathrm{Ce}$ lessivage accru aurait pour origine le dépôt humide d'acides et de $\mathrm{NH}_{4}{ }^{+}$(pluie et brouillard), et il serait stimulé par les effets de l'ozone sur les perméabilités cuticulaire et membranaire (Gunthardt-Goerg, Keller, 1987; Adams et al, 1990; Barnes, Brown, 1990). L'enrichissement des eaux de pluviolessivats résulterait cependant en partie des échanges au niveau foliaire et en partie du lessivage du dépôt sec sur la frondaison (Lindberg et al, 1986; Percy, 1989; Puckett, 1990). Cet échange d'éléments au niveau foliaire et la réabsorption des cations perdus transfèrent l'acidité à la rhizosphère.

Mais actuellement il est de plus en plus envisagé que les carences minérales foliaires seraient induites par une mauvaise alimentation minérale à partir des racines ou d'éléments non disponibles dans le sol. Les dépôts acides au niveau du sol provoqueraient un lessivage superficiel des éléments minéraux et une libération de l'aiuminium (Blank et al, 1988; Schulze, 1989; Ulrich, 1990).

Pour rechercher l'origine et évaluer l'importance de ces carences foliaires aux niveaux histologique et cellulaire, nous avons entrepris une étude par microanalyse $X$.

Si en rapport avec le dépérissement forestier, de nombreuses analyses foliaires 
ont été effectuées, en revanche les études à l'échelle cellulaire de la composition minérale des feuilles ont été peu abordées. Des profils des concentrations en Ca et $\mathrm{K}$ dans quelques stomates d'aiguilles d'épicéa ont été montrés par Bosch et al (1983). Les travaux récents de Fink (1990) sur des arbres dépérissants in situ et sur des arbres traités par des "pluies acides» montrent des accumulations extracellulaires de cristaux d'oxalate de calcium au niveau de l'épiderme. Ces accumulations n'existent cependant pas sur les parois externes des cellules épidermiques, et ceci serait expliqué par le phénomène du pluviolessivage du calcium.

Le but de nos recherches par microanalyse $\mathrm{X}$ est quadruple; il consiste à :

- vérifier l'existence de perturbations minérales dans les feuilles vertes, préalables au développement de symptômes visibles;

- déterminer les tissus les plus touchés par ces perturbations et estimer les conséquences physiologiques qui en découlent;

- obtenir, grâce à la microlocalisation des perturbations ioniques, des indications sur les facteurs à l'origine du dépérissement (effets de facteurs atmosphériques qui altéreraient les tissus périphériques des feuilles, ou effets de facteurs édaphiques qui affecteraient les tissus centraux (Fink, 1988; Zoettl et al, 1989; Huettl et al, 1990);

- comparer les modifications minérales liées aux facteurs du dépérissement avec celles induites par le vieillissement des aiguilles.

\section{MATÉRIEL ET MÉTHODES}

\section{Matériel végétal}

Des rameaux ont été prélevés en octobre au Col du Donon (Vosges) sur des épicéas âgés de 80 à 120 ans, de 2 classes extrêmes de dépérissement (classe 0 : $0-10 \%$ de chute d'aiguilles, arbres sains et classe II : $25-60 \%$ de chute d'aiguilles sans jaunissement).

Les prélèvement ont été effectués par «tir au fusil', dans le tiers supérieur des houppiers. Les rameaux ont été immédiatement recoupés sous l'eau. Au laboratoire, après une nuit à l'obscurité de façon à obtenir un maximum de fermeture des stomates, les rameaux ont été plongés dans l'azote liquide, puis les aiguilles vertes sont séparées par année d'âge (uniquement les aiguilles de l'année et celles de 3 ans ont été retenues) et stockées au congélateur à $-70^{\circ} \mathrm{C}$.

Ces prélèvements ont porté sur 2 classes de dépérissement $\times 3$ arbres par classe $\times 2$ années d'aiguilles par branche.

\section{Préparation des échantillons et analyses}

Des coupes transversales (épaisseur $500 \mu \mathrm{m}$ ) ont été effectuées dans les aiguilles, au moyen d'un cryomicrotome à $-30^{\circ} \mathrm{C}$. Les coupes ont été ensuite lyophilisées à $-10^{\circ} \mathrm{C}$, de façon à éviter le déplacement des ions. Avant la microanalyse $X$ (microscope électronique à balayage Cambridge-Stereoscan 90 équipé d'une diode), les coupes ont été recouvertes d'un film de carbone $(20 \mathrm{~nm})$ et fixées sur un porte-objet au moyen d'une colle conductrice au carbone (Garrec, 1983).

Les conditions de fonctionnement de la microsonde sont les suivantes :

- tension d'accélération du faisceau d'électrons : $25 \mathrm{kV}$;

-2 grossissements ont été utilisés :

* x 1520 : la surface de la zone analysée par balayage est dans ces conditions de 22,4 x 17,6 $=394 \mu \mathrm{m}^{2}$

* $\times 323$ : la surface analysée est de $91,6 \times 75=$ $6875 \mu \mathrm{m}^{2}$

- durée de l'analyse : $100 \mathrm{~s}$.

Les rayons $X$ émis par les échantillons sont détectés par une diode (analyse en dispersion d'énergie) et un programme ZAF définit les concentrations apparentes des éléments $\mathrm{K}, \mathrm{Ca}$, $\mathrm{Mg}, \mathrm{P}, \mathrm{S}, \mathrm{Mn}$ et $\mathrm{Cl}$. 


\section{Microlocalisations}

Dans les coupes transversales effectuées au milieu des aiguilles, les concentrations en ions $\mathrm{K}, \mathrm{Ca}, \mathrm{Mg}, \mathrm{Mn}, \mathrm{Cl}$, Si et $\mathrm{P}$ ont été étudiées dans 4 zones précises de tissus (fig 1 ) :

- les cellules de garde des stomates $(G=x$ $1520)$;

- l'épiderme ( $G=\times 1520)$;

- le parenchyme $(G=\times 323)$;

- les faisceaux libéro-ligneux $(G=x 323)$.

Sur les coupes lyophilisées, la distinction entre les faisceaux libériens et les faisceaux ligneux est rendue très difficile.

Ces analyses ont été répétées sur 5 aiguilles différentes.

\section{Expression des résultats}

Pour quantifier les résultats et pour connaître la relation qui transforme les concentrations apparentes en unités de concentration classiques, des témoins ont été réalisés en imprégnant des disques de papier filtre avec des concentrations croissantes et connues d'éléments. Ces témoins ont été congelés, lyophilisés et analysés de la même manière que les échantillons végétaux.

Pour chaque élément, à partir des concentrations apparentes trouvées dans les échantillons biologiques, et par comparaison avec la courbe d'étalonnage obtenue avec les témoins, la concentration réelle dans les différents tissus végétaux a été calculée (Garrec et al, 1983).

L'erreur standard $(P=0,05)$ sur la moyenne des 30 mesures ( 3 arbres $\times 2$ branches $\times 5$ ai-

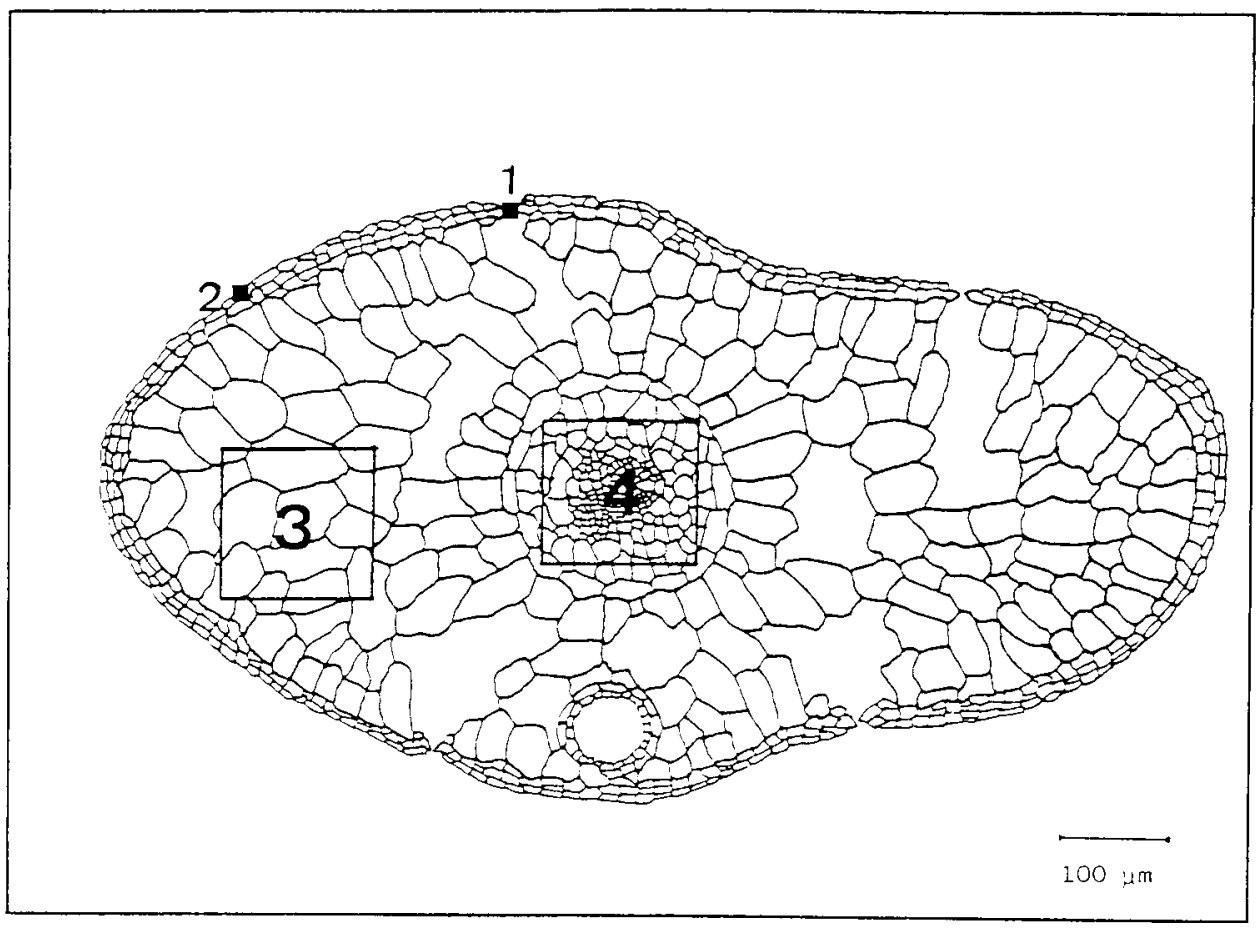

Fig 1. Dessin d'après plusieurs photographies d'une coupe idéale dans une aiguilie d'épicéa commun. Zones de micro-analyse : 1 Stomate; 2 Épiderme; 3 Parenchyme; 4 Faisceaux libéro-ligneux. 
guilles) effectuées pour chacun des 16 niveaux d'observation ( 2 classes $\times 2$ âges $\times 4$ zones) est :

$\mathrm{P}=18 \% ; \mathrm{Cl}=14 \% ; \mathrm{K}=19 \% ; \mathrm{Ca}=29 \%$ et $\mathrm{Mn}=$ $8 \%$.

Une réduction des données et une analyse de la variance à 3 critères de classification (classe, âge, zone) ont été réalisées par le programme PROC GLM de SAS à partir des paramètres mesurés: $\mathrm{K}, \mathrm{Ca}, \mathrm{P}, \mathrm{Cl}, \mathrm{Mn}$.

\section{RÉSULTATS}

Les figures 2, 3 et 4 représentent respectivement les variations des concentrations en $\mathrm{K}$, en $\mathrm{Ca}$ et en $\mathrm{Mn}$ dans les différents tissus foliaires, soit en fonction de l'âge (toutes classes de dépérissement confondues), soit en fonction de la classe de dépérissement (tous âges confondus).

Dans le tableau I, nous avons indiqué les variations en pourcentage des concen- trations des éléments $\mathrm{K}, \mathrm{Ca}$ et $\mathrm{Mn}$ en fonction de l'âge des aiguilles, ou de leur niveau de dépérissement, et ceci pour les différents tissus foliaires analysés.

Dans le tableau II, nous avons récapitulé les résultats d'analyses de variances.

Des 3 critères de classification pris en compte, la zone de tissus analysée est la plus déterminante pour tous les éléments. Ceci confirme que les éléments minéraux ont des sites de localisation préférentiels dans les tissus végétaux.

Tous les éléments minéraux, à l'exception des teneurs en $\mathrm{Ca}$, permettent de distinguer l'âge des aiguilles. La classe de dépérissement est associée à des différences au moins hautement significatives pour les teneurs en $\mathrm{K}, \mathrm{Mn}$ et $\mathrm{P}$.

Nous indiquons ci-dessous, élément par élément, les résultats de cette analyse statistique.

Tableau I. Variations en \% de $\mathrm{K}, \mathrm{Ca}$ et $\mathrm{Mn}$ dans les différents tissus d'aiguilles au cours de leur vieillissement ou de leur dépérissement.

\begin{tabular}{lcccc}
\hline & Stomates & Épiderme & Parenchyme & $\begin{array}{c}\text { Faisceaux } \\
\text { libero-ligneux }\end{array}$ \\
\hline $\begin{array}{l}\text { Age } \\
1 \text { à 3 ans }\end{array}$ & $-45 \%$ & $-46 \%$ & $-55 \%$ & $-65 \%$ \\
$\begin{array}{l}\text { Dépérissement } \\
\text { Classe 0 à II }\end{array}$ & $-37 \%$ & $-42 \%$ & $-21 \%$ & 0 \\
$\begin{array}{l}\text { Age } \\
1 \text { à 3 ans }\end{array}$ & $+29 \%$ & $+66 \%$ & $+106 \%$ & $+22.2 \%$ \\
$\begin{array}{l}\text { Dépérissement } \\
\text { Classe } 0 \text { à II }\end{array}$ & $+42 \%$ & $+15 \%$ & $+11 \%$ & $+11 \%$ \\
$\begin{array}{l}\text { Age } \\
1 \text { à } 3 \text { ans } \\
\text { Dépérissement } \\
\text { Classe } 0 \text { à II }\end{array}$ & $+1030 \%$ & $+505 \%$ & $+104 \%$ & $+135 \%$ \\
\hline
\end{tabular}


Tableau II. Analyse de la variance sur les concentration de $\mathrm{K}, \mathrm{Ca}, \mathrm{Mn}, \mathrm{Cl}$ et $\mathrm{K}$ mesurées dans les différentes zones de tissus, selon l'âge des aiguilles et la classe de dépérissement.

\begin{tabular}{|c|c|c|c|c|c|c|c|}
\hline $\begin{array}{c}\text { Source } \\
\text { de } \\
\text { variation }\end{array}$ & Classe & $\hat{A} g e$ & Tissus & $\begin{array}{c}\text { Classe } \\
x \text { Âge }\end{array}$ & $\begin{array}{r}\text { Classe } \\
\times \text { Tissus }\end{array}$ & $\begin{array}{l}\text { Tissus } \\
x \text { Âge }\end{array}$ & $\begin{array}{c}\text { Classe } \\
x \text { Tissus } \\
x \text { Âge }\end{array}$ \\
\hline K & $X X X$ & $X X X$ & $x X X$ & NS & $X X X$ & $X X X$ & NS \\
\hline $\mathrm{Ca}$ & NS & NS & $X X X$ & NS & $X X$ & $X X X$ & NS \\
\hline $\mathrm{Mn}$ & $x \times X$ & $X X X$ & $X X X$ & NS & $X X X$ & $x$ & $x$ \\
\hline $\mathrm{Cl}$ & NS & $x \times x$ & $X X X$ & NS & NS & $x x x$ & NS \\
\hline$P$ & $x x$ & $x \times x$ & $X X X$ & NS & $X X X$ & $x \times X$ & NS \\
\hline
\end{tabular}

NS : non significatif; $X$ : significatif $(P<0,05) ; \mathrm{XX}$ : hautement significatif $(P<0,01) ; \mathrm{XXX}$ : très hautement significatif $(P<0,001)$.

\section{Microlocalisation du potassium (fig 2)}

Les différences de concentrations en $K$ sont très hautement significatives pour les 3 critères de classification (tableau II).

Nous constatons que :

POTASSIUM

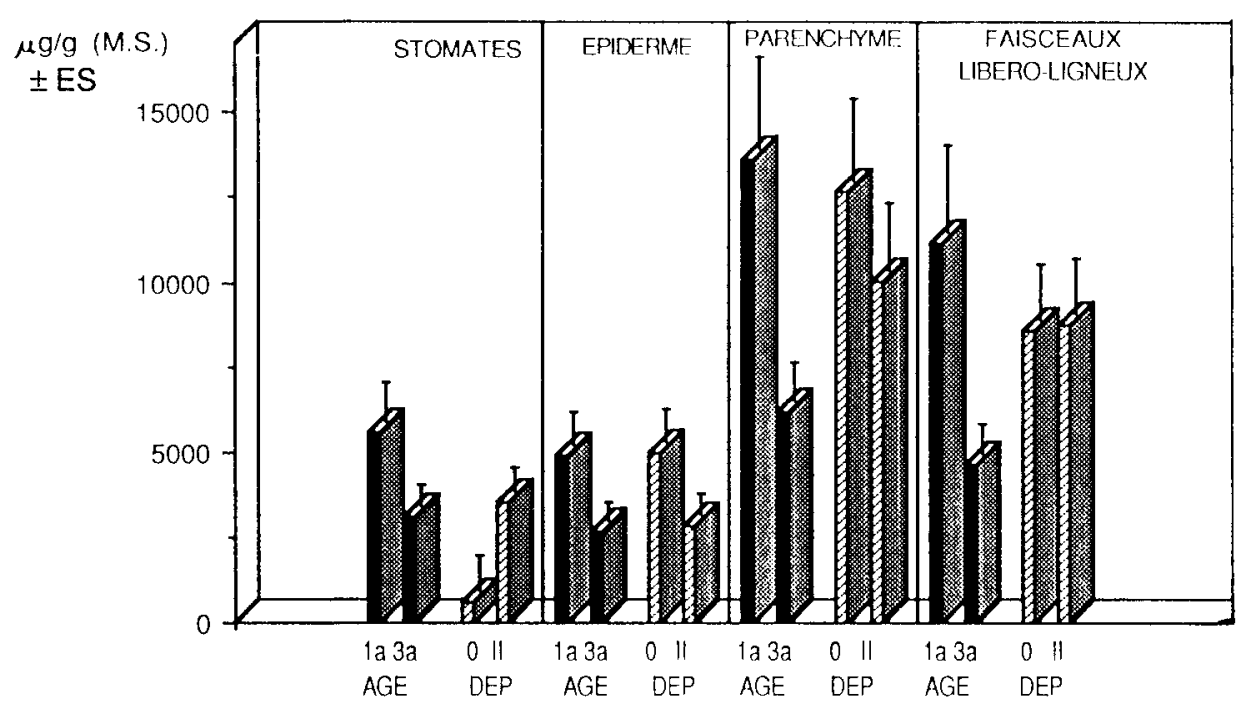

Fig 2. Variations des concentrations en potassium dans les différents tissus d'aiguilles vertes d'épi-

céas en fonction de l'âge de celles-ci de leur niveau de dépérissement.
- les concentrations de $\mathrm{K}$ dans l'épiderme et dans les stomates sont toujours nettement inférieures à celles du parenchyme et des faisceaux libéro-ligneux (rapport de l'ordre de 1/2);

- le vieillissement entraîne dans tous les tissus une baisse des concentrations en $\mathrm{K}$; 
celle-ci est particulièrement accentuée au niveau du parenchyme et des faisceaux (tableau I);

- le niveau croissant de dépérissement est associé dans la majorité des tissus à une baisse des concentrations en $\mathrm{K}$. Celle-ci est importante au niveau de l'épiderme et des stomates (tableau I), mais elle ne s'observe plus dans les faisceaux libéroligneux.

\section{Microlocalisation du calcium (fig 3)}

Comme pour le $\mathrm{K}$, les concentrations en Ca dans l'épiderme et dans les stomates sont inférieures à celles du parenchyme et des faisceaux conducteurs (rapport de l'ordre de 1/2; variations très hautement significatives entre les différentes zones de tissus) (tableau II).

Le vieillissement se caractérise dans les tissus par une augmentation non significa- tive des concentrations en $\mathrm{Ca}$, surtout au niveau du parenchyme et des faisceaux conducteurs (tableau I). La micro-analyse, comme les résultats d'analyses foliaires classiques, montrent un enrichissement plus ou moins marqué en $\mathrm{Ca}$ et un appauvrissement en $\mathrm{K}$ avec l'âge.

Le dépérissement a pour conséquence une augmentation non significative des concentrations en $\mathrm{Ca}$, plus accentuée au niveau de l'épiderme et des stomates (tableau I).

\section{Microlocalisation du manganèse (fig 4)}

Le manganèse est un élément très discriminant (tableau II).

Comme pour le $\mathrm{K}$ et le $\mathrm{Ca}$, les concentrations les plus fortes en Mn s'observent dans le parenchyme et les faisceaux conducteurs (envrion 3 fois plus que dans l'épiderme et les stomates).

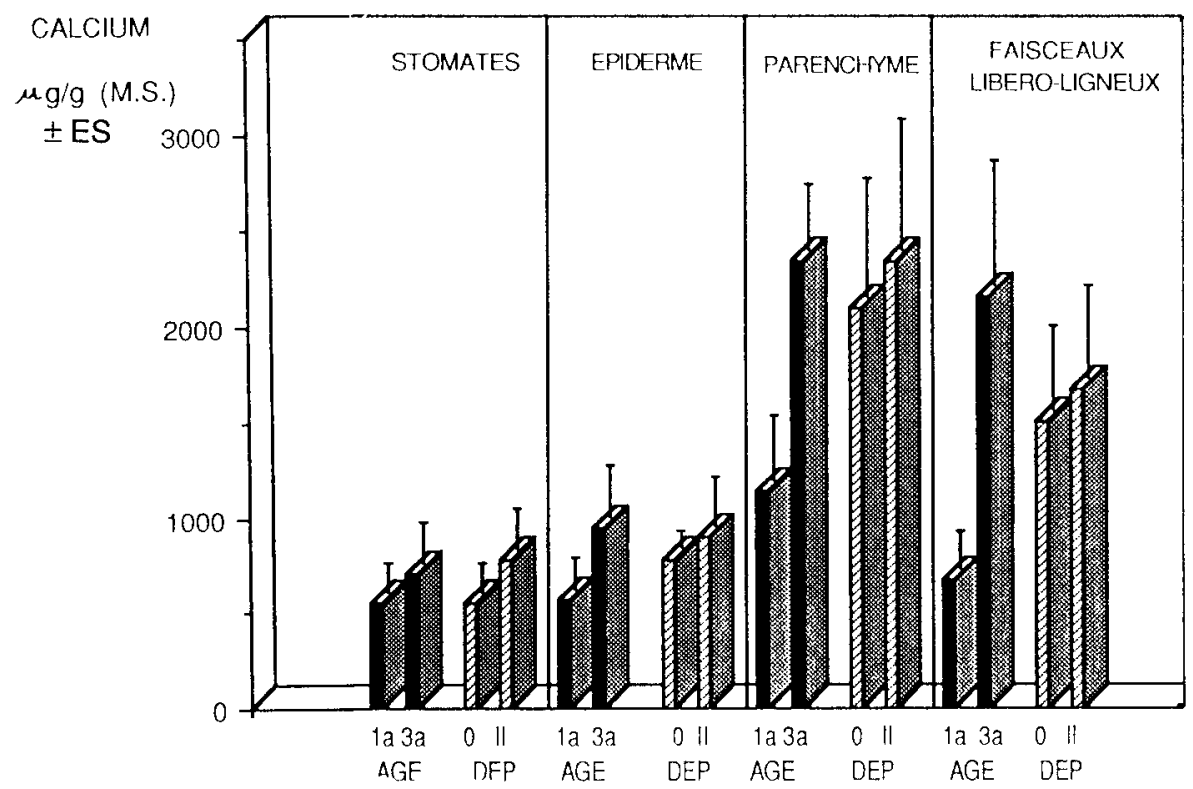

Fig 3. Variations des concentrations en calcium dans les différents tissus d'aiguilles vertes d'épicéas en fonction de l'âge de celle-ci ou de leur niveau de dépérissement. 


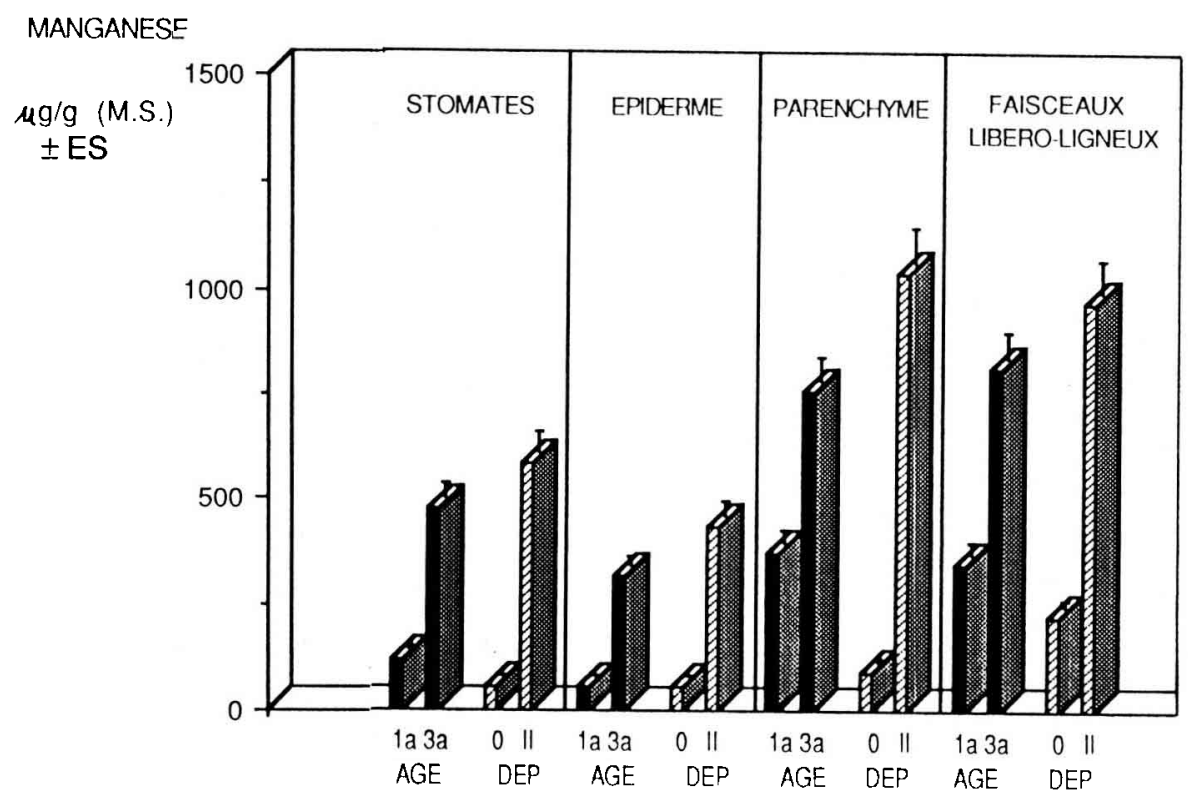

Fig 4. Variations des concentrations en manganese dans les différents tissus d'aiguilles vertes d'épicéas en fonction de l'âge de celles-ci ou de leur niveau de dépérissement.

Le vieillissement cause une très nette augmentation des concentrations en $\mathrm{Mn}$ dans tous les tissus, plus importante dans l'épiderme et les stomates (tableau I).

Le dépérissement amène également une très nette augmentation des concentrations en $\mathrm{Mn}$, légèrement plus marquée au niveau de l'épiderme et des stomates (tableau I).

\section{Microlocalisation du phosphore, du soufre, du chlore et du magnésium (fig 5)}

La figure 5 représente respectivement les variations des concentrations en $P, S, C l$ et $\mathrm{Mg}$ en fonction de l'âge des aiguilles (toutes classes de dépérissement et tous tissus confondus), ou en fonction de la classe de dépérissement (tous âges et tous tissus confondus).

A l'exception du phosphore et du chlore, les concentrations en $\mathrm{S}$, et surtout en $\mathrm{Mg}$ sont faibles, et sont à la limite de détection par la microsonde. Les variations de $P$ et de $\mathrm{Cl}$ observées entre zones de tissus ou en fonction de l'âge sont très hautement significatives (tableau II). Le vieillissement est associé à une baisse des concentrations en $\mathrm{P}(43 \%)$ et $\mathrm{Cl}(80 \%)$, mais en revanche le dépérissement entraîne une hausse très significative des teneurs en $P$ et une baisse non significative du $\mathrm{Cl}$.

\section{DISCUSSION}

L'étude par microanalyse d'un certain nombre d'éléments minéraux dans les tis- 


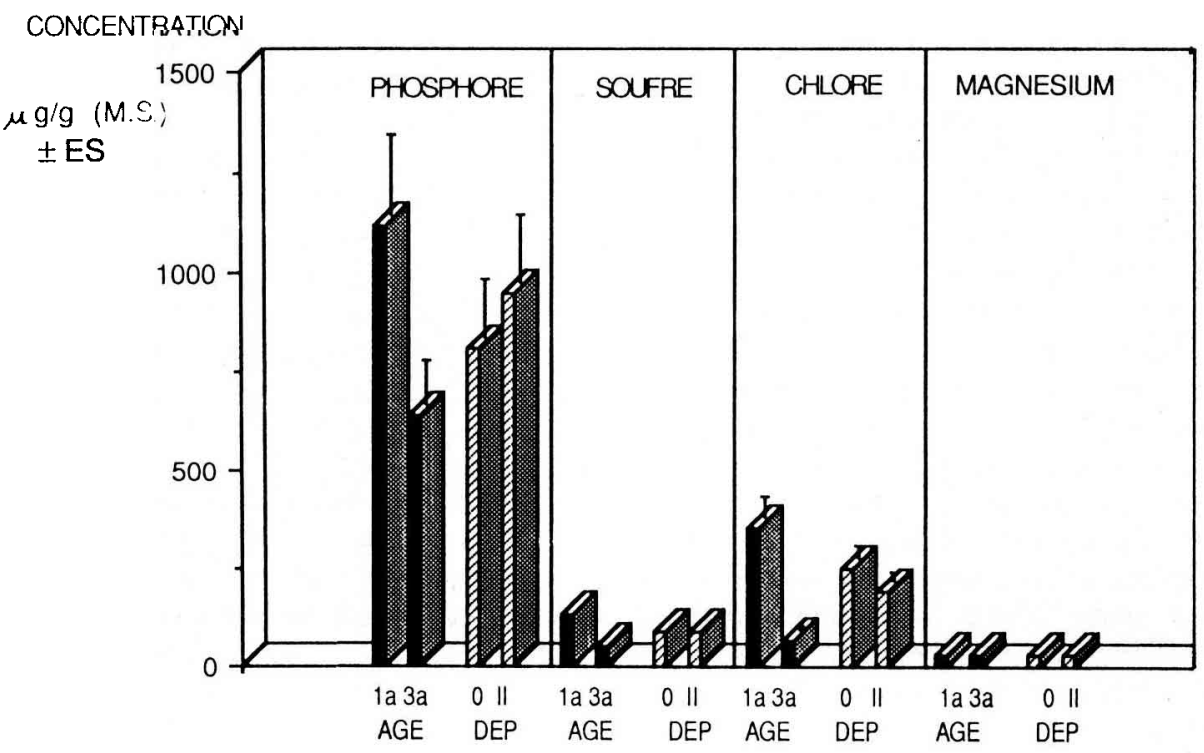

Fig 5. Variations des concentrations en $\mathrm{P}, \mathrm{S}, \mathrm{Cl}$ et $\mathrm{Mg}$ dans les aiguilles vertes d'épicéas en fonction de l'âge de celles-ci ou de leur niveau de dépérissement.

sus d'aiguilles d'épicéas montre que si les facteurs du dépérissement entraînent des variations de concentration, celles-ci sont limitées tant que les aiguilles restent vertes. II semble donc, d'après nos résultats, que les carences minérales ne soient pas les causes primaires du dépérissement, tout au moins dans le cas d'arbres défeuillés sans jaunissement.

Dans les aiguilles saines ou dépérissantes du Donon, les teneurs en Mg sont particulièrement basses, et les teneurs en $\mathrm{Mn}$ sont particulièrement élevées. Ces observations sont à mettre en relation avec les caractéristiques des sols locaux, qui sont connus pour être pauvres en $\mathrm{Mg}$ (Jover et Barneoud, 1978) et riches en $\mathrm{Mn}$ (proximité de formations dévoniennes; Bonneau, communication personnelle). Peut-être faut-il voir dans ces concentrations anormales un facteur prédisposant au dépérissement pour les épicéas de cette région?
Le principal acquis de ce travail est d'avoir montré le parallélisme entre les effets du vieillissement et ceux des facteurs du dépérissement. En effet, pour ces 2 phénomènes, les variations de concentration tissulaires et cellulaires évoluent toujours dans le même sens.

Cette similitude, que nous constatons par analyse élémentaire, confirme que les facteurs atmosphériques à l'origine de ce dépérissement agissent à un niveau faible. En effet, les travaux de Pierre (1984) ont montré que l'impact de stress faibles se répercutait, avant tout, par la mise en route d'un processus de sénescence précoce.

Toutefois, dans le détail les mécanismes associés au dépérissement et au vieillissement sont différents, en particulier pour les éléments $\mathrm{K}$ et $\mathrm{Ca}$ : l'effet des facteurs du dépérissement est plus marqué au niveau des cellules stomatiques et épidermiques. Le vieillissement est quant à lui 
associé à de fortes modifications de composition élémentaire, au niveau du parenchyme et des faisceaux conducteurs. Des résultats similaires ont été obtenus par Eschrich et al (1988), dans le cadre d'une étude par microanalyse $X$ de la répartition des éléments minéraux dans les feuilles de hêtre au cours de leur sénescence.

Cela confirme donc que le vieillissement est associé surtout à des perturbations minérales au niveau des tissus centraux des aiguilles, en particulier au niveau des faisceaux libéro-ligneux (Fink, 1988; Schmitt, Ruetze, 1989). En revanche, le dépérissement aurait pour conséquence des perturbations minérales localisées dans les tissus périphériques des aiguilles, par suite de la lixivation foliaire, par exemple.

Ces perturbations importantes, localisées exclusivement dans les tissus périphériques, laissent penser que, dans les arbres dépérissants à aiguilles vertes, ce phénomène résulterait avant tout de l'action de facteurs atmosphériques (polluants gazeux et précipitations atmosphériques). Des conclusions similaires ont été formulées en microscopie optique et électronique, dans le cadre d'études sur les altérations des structures au niveau cytologique, dans les aiguilles d'arbres dépérissants (Fink, 1988; Forschner et al, 1989).

Ces résultats suggèrent aussi une relation entre ces perturbations minérales et l'augmentation de concentration ionique des pluvio-lessivats, classiquement observée sous les houppiers d'arbres dépérissants. Du point de vue des bilans, les pertes en ions par les tissus foliaires sont toujours très faibles, comparativement aux stocks d'éléments foliaires (Abrahamsen, 1980; Amthor, 1986; Joslin et al, 1988).

Sur le plan physiologique, ces perturbations ioniques dans les tissus périphériques des aiguilles, en modifiant en particu- lier les concentrations en $\mathrm{K}$, doivent altérer le fonctionnement des stomates (Laffray et al, 1982), et intervenir en partie dans les déficits hydriques que l'on constate dans les aiguilles d'arbres dépérissants (Badot et al, 1988; Rosenkranz et al, 1989).

D'une façon générale, nos résultats semblent indiquer, pour expliquer ces phénomènes de dépérissement forestier, que lorsque l'arbre est en "équilibre» avec son sol (cas d'un sol peu carencé ou d'une faible demande en éléments par l'arbre), ce qui se manifeste visuellement par des aiguilles vertes, les stress liés au dépérissement (sécheresse, pollution atmosphérique) n'entraînent dans ces conditions que des perturbations faibles au niveau des aiguilles. Celles-ci se caractérisent avant tout par la mise en route d'un processus de sénescence précoce (chute d'aiguilles).

En revanche, lorsque l'arbre est en "déséquilibre» avec son sol (cas d'un sol pauvre en éléments nutritifs et plus ou moins carencé par les dépôts acides et/ou d'une demande trop forte en éléments de la part de l'arbre), ceci se traduit alors par des carences marquées au niveau des aiguilles, et par leur jaunissement. Si l'impact direct de la pollution atmosphérique sur les relations ioniques dans les aiguilles est alors comparativement peu détectable, il ne faut pas oublier son impact via le sol.

\section{Remarques}

Dans le cadre d'études par microanalyse, il est toujours très difficile de comparer les concentrations en éléments obtenues par cette méthode, à celles obtenues par les méthodes courantes. En effet, par cette technique, seules quelques cellules d'un tissu particulier sont prises en compte lors de chaque analyse, et de ce fait, les valeurs peuvent être différentes (d'un rapport 
1 à 4) de celles obtenues par les analyses foliaires classiques qui concernent une moyenne sur l'ensemble des tissus.

Signalons également que si la microanalyse $X$ est une méthode très précise, en revanche elle n'est pas très sensible. En particulier lorsque les concentrations des éléments légers se situent au-dessous de $100 \mu \mathrm{g} / \mathrm{g}$, les résultats ne peuvent plus être donnés qu'à titre indicatif. C'est le cas, dans cette étude, pour le magnésium et le soufre. Nous les avons cependant représentés sur les figures, afin de signaler leurs faibles teneurs relatives, en sachant que les valeurs fournies par l'appareil sont entâchées d'une trop grande erreur pour être prises en considération.

Cette technique n'a donc pas permis d'aborder la répartition du magnésium, bien que cet élément soit connu pour jouer souvent un rôle important dans le phénomène du dépérissement.

\section{RÉFÉRENCES}

Abrahamsen G (1980) Acid precipitation, plant nutrient and forest growth. Proc int Conf Ecol Impact Acid Precip, Norway, SNSF project (contribution FA 65/80)

Adams CM, Caporn SJM, Hutchinson TC (1990) Crystal occurence and wax disruption on leaf surfaces of cabbage treated with simulated acid rain. New Phytol 114, 147-158

Amthor JS (1986) An estimate of the "cost" of nutrient leaching from forest canopies by rain. New Phytol 102, 359-364

Badot PM, Garrec JP, Millet B, Badot MJ, Mercier $J$ (1988) Dépérissement et état hydrique des aiguilles chez le Picea abies. Can J Bot 66, 1693-1701

Barnes JD, Brown KA (1990) The influence of ozone and acid mist on the amount and wettability of the surface waxes in Norway spruce (Picea abies (L) Karst). New Phytol 114, 531-535

Becker $M$ (1989) The role of climate on present and past vitality of Silver fir forests in the
Vosges mountains of northeastern France. Can J For Res 19, 1110-1117

Blank LW, Roberts TM, Skeffington RA (1988) New perspectives on forest decline. Nature 336, 27-30

Bosch C, Pfannkuch E, Rehfuess KE (1983) Uber die Erkrankung der Fichte (Picea abies Karst) in den Hochlagen des Bayerischen Waldes. Forstwiss Centralbl (Hamb) 102, 167-181

Eschrich W, Fromm J, Essiamah S (1988) Mineral partitioning in the phloem during autumn senescence of beech leaves. Trees 2, 78-83

Fink S (1988) Histological and cytological changes caused by air pollutants and other abiotic factors. Air pollution and plant metabolism (S Schulte-Hostede, NM Darrall, LW Blank, AR Wellburn, eds) Elsevier Applied Science, Amsterdam 36-54

Fink S (1990) Coupling water and nutrient movement in healthy and damaged conifer needles: a study in physiological anatomy. Conference Abstracts "Acidic Deposition". Its nature and impacts". Glasgow, 16-21 septembre, 47

Forschner W, Schmitt V, Wild A (1989) Investigations on the starch content and ultrastructure of spruce needles relative to occurrence of novel forest decline. Bot Acta 102, 181260

Garrec JP (1983) Microanalyse $X$ des éléments diffusibles en biologie. Microanalyse $X$ en biologie (S Quintana, S Hapern, eds). Société française de microscopie électronique, Paris, 141-150

Garrec JP, Vavasseur A, Michalowicz G, Laffray D (1983) Stomatal movements and repartition of the elements $\mathrm{K}, \mathrm{Cl}, \mathrm{Na}, \mathrm{P}, \mathrm{Ca}, \mathrm{Mg}$ and $S$ in the stomatal complexes of Vicia faba and Commelina communis. Electron probe studies. Z Pflanzenphysiol 112, 35-42

Gunthardt-Goerg MS, Keller T (1987) Some effects of long term ozone fumigation on Norway spruce. II. Epicuticular wax and stomata. Trees 1, 145-150

Huettl RF (1989) Liming and fertilization as mitigation tools in declining forest ecosystems. Water Air Soil Pollut 44, 93-118

Huettl RF, Fink S, Lutz HJ, Poth M, Wisniewski $J(1990)$ Forest decline, nutrient supply and diagnostic fertilization in southwestern Ger- 
many and in southern California. For Ecol Manage 30, 341-350

Jover J, Barneoud C (1978) Carence magnésienne sur épicéa commun. Ann AFOCEL 2, 443-446

Joslin JD, McDuffie C, Brewer PF (1988) Acidic cloud water and cation loss from Red spruce foliage. Water Air Soil Pollut 39, 355-363

Laffray D, Louguet P, Garrec JP (1982) Microanalytical studies of potassium and chloride fluxes and stomatal movements of two species: Vicia faba and Pelargonium $X$ hortorum. J Exp Bot 33, 771-782

Landmann G, Bonneau M, Adrian M (1987) Le dépérissement du sapin pectiné et de l'épicéa commun dans le massif vosgien est-il en relation avec l'état nutritionnel des peuplements ? Rev For Fr 39, 5-11

Lindberg SE, Lovett GM, Richter DD, Johnson DW (1986) Atmospheric deposition and canopy interactions of major ions in a forest. Science 231, 141-145

Lovett GM, Lindberg SE, Richter DD, Johnson DW (1985) The effects of acidic deposition on cation leaching from three deciduous forest canopies. Can J For Res 15, 1055-1060

Percy KE (1989) Vegetation, soils and ion transfer through the forest canopy in two Nova Scotia lake basins. Water Air Soil Pollut 46, 73-86

Pierre M (1984) Effets non visibles de la pollution des végétaux par le $\mathrm{SO}_{2}$ : modifications du métabolisme et coût physiologique chez le haricot (Phaseolus vulgaris). Thèse de Doctorat ès Sciences. Université Pierre et Maris Curie, Paris

Puckett LJ (1990) Estimates of ion sources in deciduous and coniferous throughfall. Atmos Environ 24 A, 545-555
Rehfuess KE (1987) Perceptions on forest diseases in Central Europe. Forestry 60, 1-11

Reynolds B, Cape JN, Paterson IS (1989) A comparison of element fluxes in throughfall beneath Larch and Stika spruce at two contrasting sites in the United Kingdom. Forestry 62, 29-39

Roberts TM, Skeffington RA, Blank LW (1989) Causes of type 1 Spruce decline in Europe. Forestry 62, 179-222

Rosenkranz T, Bareis C, Braun J, Wild A (1989) Investigation on the water status of Spruce trees in relation to the occurence of novel forest decline. Plant Physiol Biochem 27, 333340

Schmitt U, Ruetze M (1989) The sieve cells in Spruce needles (Picea abies $L$ Karst). Indicators for a disorder in assimilate translocation? Angew Bot 63, 525-532

Schulze ED (1989) Air pollution and forest decline in a Spruce (Picea abies) For Sci 244, 776-783

Sigmon JT, Gilliam FS, Partin ME (1989) Precipitation and throughfall chemistry for a montane hardwood forest ecosystem: potential contributions from cloud water. Can J For Res 19, 1240-1247

Ulrich B (1990) Effects of acidic precipitation on forest ecosystems in Europe. Acidic precipitation. Volum 2. Biological and ecological effects (DC Adriano and $\mathrm{AH}$ Johnson, eds). Springer-Verlag, Berlin, 189-272

Zoettl HW, Huettl RF (1986) Nutrient supply and forest decline in southwest Germany. Water Air Soil Pollut 31, 449-462

Zoettl HW, Huettl RF, Fink S, Tomlinson GH, Wisniewski J (1989) Nutritional disturbances and histological changes in declining forests. Water Air Soil Pollut 48, 87-109 